9. Cfr. C. Offe: Strukturprobleme des kapitatistischen Staates, Francfort, Suhrkamp, 1972 y J. Habermas: Problemas de lezitimacion en el capitalismo tardro, Buenos Aires, Amorrortu, 1975. El prolijo debate entre Habermas y Luhmann ha quedado parcialmente recogida en Theorie der GeseIschaft oder Sozialtechnologie - Was leistet die Systemforschung?, Francfort, Suhrkamp, 1975.

10. C. Offe, op. cit., p. 50.

11. J. Habermas; op. cit., p. 249.

12. Sobre este tema cfr. fundamentalmente S, Brittan: "The Economic Contradictions of DeJocracy», en British Joumal of Political Science, 5 (1975) pp. 129.159; R. Rose: "Overloaded Government" en European Studies Newsletter (1975); W. Hemis, P. Graf Kielmannsegg y U. Matz (eds.): Regierbakket, Stutgart, Kohlhammer, 1977-1979 y C. Offe: "Unregierbarkeit". Zur Renaissance konservativer Krisentheorien, en J. Habermas (ed): Stichworte zur 'Geistigen Situation der Zeit' (Bd. 1) Francfort, Suhrkamp, 1979, pp. 294-318.

13. Cfr., por ejemplo, A. Wolfe: Los limites de la legitimidad, México, Siglo XXI, 1980.

14. Son de resaltar por su interés los que aparecen en las recopilaciones de D. Held (ed.): States \& Societies, Oxford, Basil Blackwell, 1983 y M. Dogan (ed.): Comparing pluralist Democracies. Strains on Legitimacy, Boulder, Westview Press, 1988. Destaca también, por su originalidad, la aplicacion de los esquemas de Habermas y offe a
Los países del Este europeo llevada a cabo por Andrew Arato. Cfr, «Critical Sociology and Authoritarian State Socialism*, en J.B. Thornpson y D. Held (eds.): Habermas: Critical Debates. Londres, MacMillan, 1982, pp, 196-218.

15. Cir. S.M. Lipset: Is there a Legitimacy Crisis?, en Micropolitics, Vol. 3, 1 (1983), pp. 1-37.

16. Al respecto cfr. $H$. KItemt: Filosofía del Estado y criterios de legitimidad, Barcelona-Caracas, Alfa, 1979; W. Connolly (ed): Legitimacy and the State, Oxford, Basil Blackwell, 1984; J. Keane: Public life and late capitalism, Cambridge, Cambridge Univ. Press, 1984 y D. Held: Models of Democracy, Cambridge, Polity Press, 1987.

17. J. Habermas op. cit., p. 243.

18. La bibliografía sobre desobediencia civil es muy abundante, pero son de destacar fundamentalmente las obras de R. Dworkin: Los derechos en serio, Barcelona, Ariel, 1984 y P. Glotz (ed.): Ziviler Ungehorsam im Rechtsstaat, Francfort, Suhrkamp, 1983.

19. Clr. B. Guggenberger y C. Offe (eds.): $A n$ der Grenzen der Mehrheitsdemoknatie, Opladen, Westdeutscher Verlag, 1984.

20. Cfr. J. Habermas: "How is Legitimacy Possible on the Basis of Legality? $y$ "On the Idea of the Rule of Law", en S.M. McMurrin (ed.): The Tanner Lectires on Human Values VIII, Salt Lake City-Cambridge, Univ. of Utah Press-Cambridge Univ. Press, 1988, pp. $219-249$ y $249-279$ respectivamente.

\title{
La recuperación analítica de la virtud clásica
}

\author{
ANDRÉS DE FRANCISCO y FERNANDO AGUIAR \\ Universidad Nacional de Educación a Distancia
}

Las líneas que siguen constituyen un comentario de la reciente e importante obra de Antoni Domènech, De la Ética a la Politica (De la razón erótica a la razón inerte), (Barcelona, Crítica, 1989). Ante un libro semejante, no sería extraño que un imaginario y despistado lector pensara que el aulor de este libro fuera más bien un equipo interdisciplinar de especialistas, pues en él se dan cita, como quien no quiere la cosa, el filósofo moral, el economista, el filólogo, el historiador de las ideas, el epistemólogo, el escritor experimentado... Pero no, el equipo se reduce a una sola persona. Nos encontramos, en efecto, ante una de las cabezas mejor amuebladas de nucstro paisaje intelectual, y no sólo del nuestro. Mas no se trata ya de meros conocimientos 
admirablemente ordenados que hubieren dado a luz una gran obra de erudición. En verdad, esta erudición no justificaría nuestro entusiasmo si a ella no le añadiéramos, como en el caso de Domènech, la originalidad de enfoque, la apuesta normativa, la imaginación teórica y el vigor de una inteligencia bien afilada. Como se puede suponer, el resultado global es explosivo. Quien piense que exageramos no tiene más que pasearse sin miedo por sus páginas: nos dará la razón.

\section{Acerca del instrumental analítico}

La teoría de juegos y la teoría de la teoría formal de la racionalidad han aportado en las últimas decadas las explicaciones más penetrantes de la conducta social. Aunque también se ha intentado -no sin éxito- su aplicación a la crítica textual en casos concretos como las teorias políticas de Rousseau y Hobbes (o textos bíblicos), nadie hasta ahora se había atrevido a reconstruir con este utillaje la historia de la racionalidad práctica occidental tout court: desde Platón hasta Habermas y Rawls. Esto es justamente lo que A. Domènech lleva a cabo admirablemente en De la Ética a la Política, marcando así un hito en la bistoria de la interpretación de textos filosóficos.

En la vida cotidiana nos vemos en la continua obligación de tomar decisiones, de elegir un determinado curso de acción. La teoría estándar de la elección racional sólo considera racional aquella acción que mejor satisface nuestros deseos y preferencias o, dicho más técnicamente, aquella acción que maximiza una función de utilidad, en la que se expresan nuestras preferencias. Cual sea el origen y contenido de esas preferencias es algo que va más allá del ámbito de dicha teoría, interesada sobre todo en la consistencia del acto mismo de decidir, o mejor dicho, en que nuesiras preferencias sean transitivas, consistentes.

Pero, como es obvio, no siempre decidimos aisladamente, sino que a menudo nuestras decisiones dependen de lo que hagan o elijan los demás, de nuestra interacción con otros. Es aquí donde interviene la teoría de juegos de estrategia formulada originariamente por $J$. von Neumann y $O$. Morgenstem para explicar la conducta de los agentes económicos. Uno de los juegos que mayor poder analítico ha demostrado es el denominado "dilema del prisioneron, profusamente empleado por Domènech en su obra. El dilema nos presenta dos jugadores con dos estrategias bien definidas, cooperar o defraudar. La búsqueda del mejor resultado individual fuerza a los jugadores a frustrar la cooperación, a cometer free niding, obtenicndo un resultado «subóptimo» para ambos: la racionalidad conduce, pues, al desastre colectivo. La lógica del dilema es implacable.

A. Domènech, además de hacer un prolijo uso del dilema del prisionero, aporta gran cantidad de juegos nuevos de su propia cosecha, convirtiendo a la teoría de juegos -como veremos- en un potentísimo instrumental analítico en el ámbito de la hermenéutica filosófica.

\section{Arquitectura del libro}

El hombre, además de creencias sobre el mundo externo, tiene deseos y preferencias, y capacidad para reflexionar sobre unos y otras. A la reflexión sobre nuestras creencias se le llama racionalidad epistémica, y racionalidad moral a la reflexión crítica sobre nuestros deseos. La tesis sustantiva del libro de Domènech es - parcialmente coincidente con la de A. Macintyre-1 ${ }^{1}$ que la cultura occidental moderna ha supues- 
to, respecto de la clásica, tanto una hipertrofia de la racionalidad epistémica, científico-técnica, como una hipotrofia de su racionalidad moral, tanto un avance gigantesco en la exploración del mundo exterior como un subdesarrollo en la exploración del mundo interior. Así, usando la terminología del autor, si la cultura moral clásica de procedencia socrática está permeada por la racionalidad "erótica» (en el sentido platónico), la racionalidad moderna es "inerte», esto es, si la primera presupone una psicología profunda (con distintos órdenes de preferencias), la segunda se levanta sobre una psicología plana (con un primer orden de preferencias indomables). Así, mientras que el individuo erótico-racionalmente guiado es capaz de elegir no sólo su mejor acción sino su mejor deseo, el individuo racionalmente inerte es víctima de sus pasiones, pues está su razón al servicio de las mismas. En otros términos, el arquetipo de hombre clásico está libre de pasiones y puede, por tanto, elegir sus deseos, al contrario que el arquetipo moderno, que está dominado por aquéllas, al considerarlas inextínguibles. Si el primero puede actuar según su mejor razón, esto es, puede elegir su mejor deseo y, por tanto, su acción es tan racional como libre; con el hombre modemo acontece más bien lo inverso. Si aquél es enkrático, éste está dominado por la inercia de sus pasiones: es akrático.

El concepto de «enkrateia» es central para el propósito del autor, que no es otro que el de "reconstruir la médula de la racionalidad "erótica" antigua y la génesis de la racionalidad "inerte" moderna a partir de la destrucción de aquella». Es central - decimos- porque permite entender lo incomprensible para la racionalidad moderna, a saber, la socrática ecuación "virtud=felicidad=libertad», esa «absurda identi- dad» con la que - según NietzscheSócrates había hechizado a la filosofía antigua y de la que ésta nunca había logrado zafarse. La «enkrateia», la "libertad interior" hace al sabio, pues le permite guiar su conducta racionalmente. $\mathrm{Y}$ por ello mismo es virtuoso el sabio, sólo él tiene "areté», sólo él uestá en forma». Mas, a su vez, sólo el sabio - por ser virtuoso- es feliz, porque -como demuestra la polisemia del verbo «práttein"- al actuar bien, le va bien.

En esta concepción libre y racional de la virtud está la clave para entender lo que Domènech denomina stangente ática», es decir, la armonía - no precisamente preestablecida - entre bien privado (agathón) y bien público (kalón). Justamente porque el clásico sabe lo que es el bien privado es capaz de cooperar al bien colectivo; porque es capaz de resolver enkráticamente el conflicto intrapsíquico que le presentan sus pasiones, asi también es capaz de resolver el conflicto interpersonal de la convivencia "política» y escapar a la lógica implacable del dilema del prisionero en la que caerán -como veremos - los individuos atomizados, akráticos, psicológicamente planos, del mundo moderno. Que ello es así queda a nuestro entender ingeniosamente mostrado por el «juego de la comunidad de enkráticos» (p. 93), que se superpone - previa modificación «erótican de las preferencias de los jugadores- a un dilema del prisionero. El ciudadano ideal de la polis cambiará sus preferencias -eligiendo cooperar- para no dejarse arrastrar por la lógica del dilema: el resultado será naturalmente óptimo.

Desde la arquitectura del libro, es central el concepto de «tangente ática». En efecto, el desarrollo de la racionalidad práctica moderna puede verse como la pérdida de la misma y 
como un intento frustrado de sustituirla por algún equivalente funcional (el soberano hobbesiano, en el caso del absolutismo), de encontrar una tangente ática burguesa (el republicanismo moderno), o de ignorarla por pensar que el mecanismo del mercado y los derechos de propiedad privada que le acompañan la hacen prescindible (liberalismo). Pero en todos estos casos asistimos a una "venganza de la virtud» clasica: el liberalismo desemboca en un absolutismo encubierto, es decir, enmascara a la soberanía pretextando embridarla mediante el Derecho, al tiempo que la tradición republicana fracasa en su intento de "restauración" de la virtud en condiciones modernas: Rousseau, a lo sumo, consigue una "tangente espartana», una fusión de bien privado y bien público en la que éste fagocita a aquél (p. 200). Por otro lado, la génesis del Estado absolutista hobbesiano, del "Gran Artificio", esto es, el surgimiento de la decisión del contrato, sólo es conceptualmente consistente si presuponemos la existencia de distintos ordenes de preferencias en los individuos $\mathrm{y}$, con ellas, su capacidad para poner en práctica estrategias de precommitment. Pero ello da al traste con la lógica de funcionamiento del modelo absolutista, que presupone una concepción privativa de la naturaleza humana, con un sólo orden de preferencias egoistas. Ahora bien, como demuestra Spinoza - corrigiendo en esto a Hobbes - la docrina absolutista presupone más bien la existencia de metapreferencias pues, sin ellas, no es posible la akrasía de los súbditos, base de su decisión de "ligarse externamente» a un soberano absoluto. Sin embargo, pese al refinamiento spinoziano de la doctrina absolutista, la virtud se venga igualmente de ella. Esto queda claro a partir de la crítica del Rousseau republicano a resultas de la cual se quiebra la lógica absolutista para producir una antinomia: "si los ciudadanos se obedecen a sí mismos, no necesitan del "gran artificio"; y si no se obedecen a sí mismos, el "gran artificio" no sólo no les libera..., sino que el mismo cuerpo político está en peligro de ruina, el "dios mortal" está condenado a muerte en la medida en que esa conducta se generalicen (p. 278).

En el largo y tortuoso camino hacia la modernidad práctica el cristianismo representa un decisivo punto de inflexión. Y ello por dos razones. En primer lugar, a él debemos el legado de una concepción radicalmente privativa, paulina, de la naturaleza humana, tan ajena al mundo clásico y tan influyente en el absolutismo moderno a través de Hobbes. El hombre viene al mundo tocado por el pecado, es una naturaleza "caída» cuya salvación se hace depender de la gracia divina. Así el arquetipo de hombre cristiano -pese a la influyente interpretación hegeliana- perderá toda idea verdadera de libertad interior, por el mero hecho de que hipostasía sus preferencias de segundo orden trasladándolas a la voluntad inescrutable de Dios. Todo ello tiene que ver con la segunda gran aportación del cristianismo, en parte anticipada por la metafísica estoica: la escisión del mundo entre un "reino natural» y un areino de la gracia». Esta aportación se aprecia bien -y de ahí la importancia del primer capítuloen el grave problema planteado por la teodicea ilustrada: el problema de las "disteleologías", del mal en un mundo paradójicamente creado por un Ser omnipotente (agens liberrimum) y bueno. Evidentemente, no podemos entrar aquí en los detalles y matices del capítulo. Señalaremos simplemente $-\mathrm{y}$ persiguiendo siempre las líneas maestras del libro- la importancia de la solución leibniziana. Leibniz, tras hacer un 
hábil quiebro metafísico por el que sitúa las constricciones a la actividad del Creador (la ananké platónica) en el intelecto divino - salvando asi la libertad de su voluntad y la contingencia del mundo-, basa su optimismo on una feliz armonía entre el reino físico de la naturaleza y el reino moral de la gracia, armonía mediante la cual éste $a b$ sorbe cosmológicamente las disteleologías de aquél. Leibniz construye asî una "cosmodicea" que justifica un optimismo sólo cosmológico, no social: su cosmodicea no implica, pues, una «sociodicea». Esta será sin embargo -piensa A. Domènech- la tentación permanente del pensamiento político moderno: construir ya un reino natural de la gracia (liberalismo) ya un reino artificial (absolutismo). De ahí la importancia arquitectónica que para el libro tiene el ajuego del reino de la gracia» leibniziano (p, 73), A su debido tiempo y no sin sorpresa, descubrirá el lector que este juego es formalmente idéntico al del reino artificial de la gracia y al «juego de la virtud rousseaunianas, lo cual permite entender no sólo la raíz cristiana del absolutismo moderno sino también el allamado "totalitarismo democrático" de la "voluntad general" de Rousseau» (p. 273).

El mundo moderno -piensa A. Domènech - intenta rescatar una tangente ética por dos vías, por la vía del imperium y por la vía de la res publica. Ello tiene que ver, una vez más, con los dos usos que la modernidad hace del cristianismo, un uso imperial y un uso escatologico. En la vá del imperium (el imperio de la ley, del derecho) se sitúan el liberalismo político y el absolutismo. La diferencia entre ambos radica en la relación que establecen entre derecho y soberania: mientras que para el absolutismo es el soberano quien otorga el derecho (que se hace positivo), con el liberalismo ocurre lo contrario; aquí es el derecho (natural) el que crea la soberanía intentando constreñirla y uembridarla» mediante su ley. Como recuerda Domènech, sin embargo, la soberanía de la teoría política liberal se reservará las decisiones sobre los «estados de excepción» (el «foederative power» de Locke), escapando así al imperio de la ley para pisar suelo absolutista. Pero los dos representantes de la tradición imperial (liberalismo y absolutismo) comparten el mismo silencio sobre el bien privado que se escinde del público: «al romper con el concepto clásico de virtud, el pensamiento político moderno no puede vincular la afirmación de esos dere. chos a nocion alguna de bien htmano; el sujeto de esos derechos es terra incognita» (p. 180). El derecho (derecho de propiedad básicamente) calla sobre la felicidad individual, tan sólo aspira a regular los conflictos de la convivencia social mediada por el mercado; esto es, aspira a un imposible: conseguir el bien público (la armonía interpersonal) sin buscar siquiera el bien privado (la armonía intrapersonal). ${ }^{2}$

Del capítulo VI (en el que Domènech sitúa a Rousseau y a Kant en la tradición republicana, absolutista y liberal respectivamente) lo más importante es sin duda el "juego de la virtud kantiana*. Este ingeniosísimo juego permite no sólo una interpretación transparente de la filosofía práctica del genio báltico sino formular, además, con precisión casi matemática lo que Domènech llama «trilema» de la filosofía práctica kantiana, esto es, el que se da entre criticismo, sintesis (de ahombre inteligible" $y$ ahombre empíricos) y Dios como postulado de la razón práctica. A partir de este trilema cabe entender (cap. VII) la filosofía clásica alemana como un intento diferenciado de darle solución. Cual si de un truco de 
prestidigitador se tratara, vemos cómo los representantes de esta cumbre del pensamiento occidental encajan en una de las posibles soluciones de la matriz del juego. Schiller y Feuerbach sacrifican el criticismo para salvar la sintesis (solución $\mathrm{C}_{1} \mathrm{C}_{2}$ ); Schopenhauer - abriendo aúm más que el propio Kant la brecha entre virtud y felicidad- arranca del fracaso del maestro en su intento de compaginar la síntesis con el criticismo y asume como punto de partida lo que para Kant es un indeseado punto de llegada, esto es, el sacrificio de la síntesis para salvar el criticismo (solución $A_{1} A_{2}$ ). Sin embargo, $y$ pese al pesimismo metafísico y a una psicología profunda heredados claramente del budismo, la raíz cristiano-protestante de su pensamiento le hará abandonar tanto el racionalismo moral de Kant como el eudemonismo racionalista del budismo. Finalmente, Fichte, Schelling y Hegel «se aferran más bien -y cada uno a su peculiarísimo modo- al postulado práctico de Dios para salvar ambas cosas (solución $\left.A_{1} C_{2}\right) »(p, 293)$. Todos ellos recurren a una "visión trágica de la historias que, actuando por el lado malo, opera el bien, sirve la síntesis en un final feliz en el que virtud, felicidad y libertad terminan coincidiendo. El propio Marx, al hacer suya esta metafísica histórica, cabe en una de las soluciones del juego, claro que -si se nos permite la expresión- haciendo a Dios bajar a la tierra, esto es, racionalizando mediante una teoría que se pretende científica aquella feliz teleología. En el alineamiento de Marx en esta tradición trágico-histórica, ve A. Domènech los restos liberales del pensamiento, por lo demás republicano, de Marx: la ilusión de que una «mecánica socials llevará a la humanidad por sí sola, sin el apoyo de la virtud, a la amonía individual y social.
EI libro se cierra con una crítica del utilitarismo y de las propuestas éticas de Rawls y Habermas, presentadas por Domènech como dos intentos dístintos de superar el teorema de imposibilidad de Arrow.

\section{¿Idealización del mundo ctásico?}

Conviene salir al paso de la supuesta idealización que A. Domènech comete de la cultura moral del mundo clási$c$, tanto más cuanto que es el propio J. Mosterín, empático prologuista del libro, quien piensa que cae víctima de ella: «me parece —escribe-que Toni tiene una visión excesivamente idealizada del mundo clásico..., y de la virtud de sus ciudadanos" (pp. 15-16). Parece, pues, como si Domènech pensara que su «construcción ideal de la tangente ática estuvicra además idealmente realizada en la polis griega. Pensamos, empero, que es ésta una falsa apariencia. De hecho, A. Domènech nunca confunde ambos niveles; antes bien, se sitúa desde el comienzo en el plano de los «constructos té́ricos" (normativos), en el plano de la "cultura filosófica» (p. 78), de la alógica de los conceptos». Como era de esperar, el autor del libro no habla de hombres reales, sino de arquetipos. Por lo demás, es el propio Domènech quien reconoce que la «reflexión política de los dos grandes arquegetas (Platón y Aristóteles) es, al menos, melancólica e idealizadora de una realidad, si alguna vez existente, en trance de extincións (pp. 82-83). Esa realidad es, naturalmente, la polis, que - como nos explica Domènech- es considerada como condición de posibilidad (en las teorías ético-políticas tanto de la Academia como del Liceo) de la tangente ática.

Por otro lado, precisamente porque Domènech está manejando constructos ideales, puede calibrar la dosis de rea- 
lismo de los mismos. Asi, al comparar a Platón con Aristóteles, observa que "La consideración de las constricciones externas hace de Aristóteles un teórico de la polis más realista que Platón. Porque la ciudad da la base material de la suficiencia...» (p. 88). Finalmente, Antoni Domenech, tras resaltar los hallazgos de la ética de procedencia socrática, no esconde su principal limitación, a saber: «el que el trazado de su tangente caiga fuera de la esfera social material; la justicia platónico-aristotélica ignora - sin duda por su raíz clasista - la vida productivas (p. 103).

El ideal (y no la idealización) de la "tangente ática", el de la armonía entre el bien privado y el público, así como la ecuación "virtud=felicidad=libertad" que lo hace posible, los encuentra Domènech en las obras de Platón y Aristóteles con sus respectivas matizaciones. Aquí el autor se limita a reconstruirlo con gran precisión $y$ originalidad mediante la teoría de juegos. Sin embargo, lo importante es el uso que Domènech hace de ese ideal que el propiamente no inventa: constituye, en efecto, cl cimiento de toda la obra; pues en él se apoya Domènech para juzgar normativamente la racionalidad práctica moderna, para juzgarla críticamente, esto es, como pérdida de to que aquel ideal representaba.

\section{Racionalidad erótica y aenkrateias: una critica}

A partir de los trabajos de los filósofos H. Frankfurt y D. Davidson, y del economista $A$. Sen, en los que se apoya Domènech para construir el concepto de racionalidad erótica, parece claro que no es posible definir el concepto de persona ni hablar de acción moral si no se consideran diversos órdenes de preferencias. ${ }^{3}$ Sin embargo, ser ca- paz de someterse a preferencias de órdenes superiores no es una condición suficiente de la conducta virtuosa, de la conducta enkrática, si no se especifica el contenido de esas preferencias. La racionalidad erótica no es sino la capacidad de elcgir el mejor deseo y, por tanto, la mejor acción. Pero «mejor" no tiene aquí carga moral necesariamente: sería, antes bien, sinonimo de «lo más adecuado para conseguir nuestros fines (sean estos los que fueren) ". Los lazos de sangre, por ejemplo, me inpiden que desee matar a mi padre, pero desearía tener el deseo de matarlo. Si lo hago, si me someto a mi deseo de segundo orden, no me convierto por ello en un individuo virtuoso (en el sentido clásico) sino en un parricida. El enkratés socrático no lo es sólo por su capacidad de someterse a metapreferencias, sino porque define moralmente sus preferencias de segundo orden, lo cual es ajeno en parte al hecho de que posea una estructura motivacional rica en órdenes de preferencias. Esto, en nuestra opinión, no queda claro del todo en la obra de Domènech. La ética clásica no se entiende sin una concepción erótica de la racionalidad, pero una racionalidad erótica no nos conduce necesariamente a la virtud socrática.

\section{La quiebra de la "tangente ática": "enkrateia" $y$ "polis"}

Que el mundo moderno se levante sobre las ruinas de la tangente ática va de suyo, permeado como está por la racionalidad inerte de individuos akráticos y habiendo archivado el ideal de la polis en los anales de la historia. Sin embargo, como observa Domènech, ya con Aristóteles se tambalean sus fundamentos para quebrarse definitivamente en el mundo helenístico con la Stoa; y ello pese a que Aristóteles toda- 
vía se apoya en la racionalidad erótica y a que los estoicos incluso la profundizan. ¿Por qué, pues, el derrumbe de la tangente ática? ¿Cuáles son las condiciones que posibilitan ese delicadísimo equilibrio entre el bien privado y el público?

Es bien sabido que el contexto histórico en que se desliza la filosofia estoica en nada recuerda ya al de la polis griega. Los estoicos se encuentran ahora en un mundo desmembrado, desbordados por un cosmos de fronteras diluidas que no les ofrece seguridad. Para resistir a ese mundo sobre el que no tiene ningún poder, el sabio estoico se vuelve sobre sí mismo, sobre su microcosmos, en busca de la felicidad individual, del «bien privado». Ello le obliga a profundizar en la libertad interior del enkratés socrático, a postular heroicamente un "tercer orden de preferencias", estrechando aún más la ecuación clásica entre virtud y conocimiento. Sin embargo, y pese a dicha profundización, el sabio estoico está fuera de la tangente ática. La razón es que esa mayor libertad y sabiduría las consigue al precio de la escisión entre ética y politica: la ética del Pórtico, marcadamente individualista, refuerza así el valor de la enkrateia para compensar la pérdida de la polis. Pero ello no es suficiente para salvar la tangente: uno de sus pilares, la ciudad, arrastra consigo - al desmoronarse- la armonía entre lo privado y lo público. La enkrateia, la racionalidad erótica no es pues suficiente para dicha armonía, ni siquiera en su versión más depurada, en su versión estoica. Pero, ces acaso necesaria? Asi parece pensarlo A. Domènech cuando ve como peligra el milagro de la tangente en manos de Aristóteles, al relajar éste la misma ecuación que los estoicos refuerzan. Aristóteles, en efecto, no sólo hace compatible cierta dosis de akrasia (de raciona- lidad "imperfectan) con la virtud, sino que acepta la posibilidad de que haya virtud para con uno mismo y no para con los demás. Sin embargo, y pese al debilitamiento de sus fundamentos seróticos", .la tangente queda a salvo en Aristóteles. ¿Cómo es ello posible? Debido a que a Aristóteles todavía le respalda la polis. Ello nos permite exIraer la siguiente conclusion: sin polis el milagro de la tangente es ni más ni menos que impensable, por muy enkráticos que sean los individuos. $\mathrm{La}$ polis no sólo hace posible la tangente ática (incluso con individuos parcialmente akráticos), sino que hace a la enkrateia más fluida, más natural, más plausible: ael enkratés de la polis -dice Domènech - vive en un contexto social altamente integrado, la inmensa mayoría de las personas con las que interactúa forman parte de su cotidianidad, la interacción con ellas es iterada, es improbable que tenga encuentros únicos con alguien; la vida pública es densa y robusta, los flujos de información entre las personas, proteicos" (p. 325, nota 45). Se entiende pues que la polis tenga primacía explicativa sobre el "milagro" de la tangente. Otra cosa es que en su ausencia el bien privado no sea posible, que sí lo es, claro que al precio del heroísmo del sabio estoico.

Esta segunda vía (la búsqueda prioritaria del bien privado en el sentido clásico, o budista) parece $\mathrm{ser}_{\text {, }}$ sin embargo, el núcleo de la apuesta normativa de $\boldsymbol{A}$. Domènech que -dicho sea en su defensa- sólo queda esbozada, sugerida. Pese a ello creemos necesario -y posible- interponer aquí unas palabras de crítica:

1. La polis es el presupuesto esencial de la amonía entre bien privado y público porque hace unaturaln la virtud de los ciudadanos, porque hace snaturalmente» posible la enkrateia. Parece- 
ría pues más realista una apuesta normativa que se dirigiera, ya no principalmente a la exploración interior, al bien privado, sino - sobre todo- a la recuperación de las condiciones "políticas» que hicieron posible el milagro de la tangente. Más aún, habría que insistir en la necesidad de ir más allá de la polis misma, siendo asf que ésta era - como recuerda Domènech-compatible con situaciones de injusticia y explotación. En otras palabras, si es verdad que unecesitamos urgentemente una cultura morals nueva (p. 337) ante los ingentes peligros a los que se enfrenta la humanidad (ecológicos, demográficos, etc.), no es menos cierto que la vieja tarea de una transformacion paralela del mundo exterior, del conjunto exterior de oportunidad -como le gusta decir al autor-, es, si cabe, más urgente.

2. Si esta observación es acertada, nos vemos obligados a constatar cierta injusticia en el tratamiento que Domènech hace de Marx. En efecto, Domènech diferencia entre un Marx «republicano" y un Marx "liberal", entre un Marx que opta por la transformación del individuo y un Marx que opta por la transformación productivista del mundo como un fin en sí mismo, en la seguridad histórico-filosófica (heredera del idealismo alemán) de que esa "producción por la producción" -que se consigue al altísimo precio de la alienación universal- servirá las condiciones (abundancia) para la felicidad y autorrealización futuras de todos los individuos. "Pero esta respuesta -explica A. Domènech- es víctima de la misma ilusión moderna que, según vimos, deslumbraba al doctrinarismo liberal: la ilusion de que puede llevarse a armonía a la humanidad, de que pueden erradicarse las estructuras sociales regidas por juegos de suma cero, con solo aumentar indefinidamente el pastel a repartir. El cuerno de la abun- dancia, aun si fuera ecológicamente posible o soportable, nunca conseguiria poner fin a esas estructuras, no haría libres a los hombres, ni tampoco felices, iguales o fraternos; a la virtud corresponde esa tarea, como sin embargo, sabía muy bien el otro Marx, el que alabó a los escritores sociales antiguos porque su "investigación nunca se pregunta qué forma de propiedad es la más productiva, la que croa más riqueza", como hacen los economistas modernos, sino "qué tipo de propiedad crea los mejores ciudadanos" (pp. 334-335).

A nosotros esta dicotomía entre un Marx srepublicano" y un Marx "liberal" nos parece fértil y original. Lo que en nuestra opinión es exagerado - a la luz de la primera observación- es que Domènech llegue a decir que el Marx liberal "ha muerto para siempre" (p. 331). Exagerado, sí, porque en Marx la pregunta "republicana" por el tipo de propiedad que crea los mejores ciudadanos (socialismo) no está dicotómicamente disociada de la pregunta por los medios para la consecución de las condiciones materiales ${ }^{4}$ que hacen posible ese tipo ideal de propiedad. Y es en la respucsta a esta ineludible pregunta estratégico-normativa donde entra la “liberal" producción por la producción propuesta por Marx, pues no debe olvidarse que - según muestra el texto de las Teorias sobre la plusvalia que cita Domènech- el verdadero fin (o contenido) de esa producción es sel superior desarrollo de la individualidad».

Ciertamente, Domènech tiene razón al considerar que el «cuerno de la abundancian no es condición suficiente para la virtud ciudadana universal (y, consecuentemente, para la universal felicidad), además de ser ecológicamente imposible. Pero, ¿acaso piensa Domènech que el socialismo es posible sin esa abundancia (reducida, si se 
quiere, a sus limites ecológicos)? Ahora bicn, si el autor responde por la negativa a esta pregunta $y$, simultáneamente, acepta que el socialismo es deseable por ser el sistema de propiedad que mejores ciudadanos crea, entonces podríamos sospcchar que Domèncch tiene puesta demasiada confianza en la virtud pues, según sus palabras anteriormente citadas, sólo a clla parece corresponder la tarea de hacer felices, iguales o fraternos a los hombres.

3. La apuesta normativa de Domènech -que sigue el trayecto que va de Socrates a Buda - nos parece radical y sugerente. Podría resumirse asi: en tanto en cuanto no sepamos qué sea el bien privado nuestros intentos de conseguir el bien público están condenados al fracaso, como queda demostrado por la historia de la racionalidad práctica moderna tal cual la reconstruye convincentemente $A$. Domènech: "Pues la cultura moderna es prepóstera; pone el carro delante de los bueyes, los medios de realización de la felicidad y de la libertad por delante de la felicidad y de la libertad. Quiere satisfacer los deseos y las necesidades de los hombres, pero no sabe en qué consisten esos deseos y esas necesidades, ni admite la posibilidad de su elección racionals (p. 337). De actierdo. Pero en un mundo como el nuestra, tan lastrado por enormes diferencias de renta y oportunidad, pensamos que la propuesta de recuperar la libertad interior, el "erotismon de la razón, puede resultar una apuesta normativa tan atractiva (incluso necesaria) como impracticable, es decir, con pocas posibilidades de realización universal. Si las necesidades básicas ${ }^{5}$ de gran parte de la población mundial no están satisfechas, tal vez fuera necesario seguir completando al Marx «republicano» con el Marx aliberal».

Lo que antecede no es más que un breve esbozo de un libro cuya riqueza difícilmente se deja reseñar. De la Ética a la Politica es una obra repleta de erudición, originalisima y fuera de lo común no sólo en nuestro ámbito filosófico sino también en el internacional. Sería una pena que pasara desapercibida. En nuestras manos está ahora abrir un debate que profundice en las líneas de investigación abiertas por el autor. Si esto se lleva a cabo será una de las pocas veces en que no nos veamos obligados a traducir debates en torno a obras de una categoria muy inferior a la de Antoni Domènech.
1. Parcialmente, porque Domènech no parte del paradigma waristotelico-tomista. como MacIntyre, sino del ideal socrático del autoconocimiento que no solo hizo suyo Aristóteles sino que fue radicalizado por la Stoa y por el budismo.

2. Este es solo un aspecto do la devastadora critica que Domènech acomete del pensamiento liberal (caps. IV y V). Con un implacable mazo analítico en mano, A. Domènech persigue sin piedad sus contradicciones, sus "lineas de replieguev, le hostiga en sus falsas soluciones para rematarlo finalmente declarando la imposibilidad misma de su enúcleo conceptual». Una critica verdaderamente magistral.

3. Nos relerimos concretamente a los articulos de A. Sen: "Choice, orderings and morality", en A. Sen: Choice, Welfare and Measurement, Oxford, Blackwell, 1982 y wLos tontos racionales\%, en F. Hahn y M. Hollis, comps.: Filosofia y teoria económica, México, F.C.E, 1986; al artículo de H. Frankfurt "Freedom of the will and the concept of per" sons, Journat of Philosophy, vol. 68 (1971), 5-20; y al articulo de D. Davidson; «How is Weakness of Will Possible? (1970), en D. Davidson, Essays on Actions and Events, Clarendon Press, Oxford, 1980. 
4. En este sentido podríamos decir que el Marx "liberal" está más cerca del realismo de Aristóteles que el propio Domenech, pues pensaria, como el griego, que la tangente ática es posible porque proporciona la base material de la existencia. ¿Por qué no pensar que Marx busca esas condiciones materiales, pero ya no en el reducido marco de la polis, sino en el de la "cosmopolts $\%$ intentando, además, emradicar las situaciones de injusticia y explotación inherentes a aquẹlla?
5. El concepto de anecesidades básicas» es difícil de definir. Pero para nuestro propósito valdtá la siguiente definición: wun individuo tiene sus necesidades básicas cuando no muere de inanición, tiene un techo mínimamente seguro donde cobijarse y abrigo regular para protegerse de las inclemencias del tiempon. Es suficiente, creemos, porque una gran parte de la población mundial se halla por debajo de esos minimos.

\title{
Para una lectura actual de la Crítica del Juicio
}

\author{
VALERIANO BOZAL \\ Universidad Complutense
}

1

Las razones para la lectura actual de la Crítica del Juicio son varias y de diversa condición. Algunas estrictamente académicas, como la influencia de esta obra sobre las reflexiones estéticas posteriores o su papel en el sistema de la filosolía kantiana, otras, y a estas deseo referirme aquí, desbordan su ámbito, a la vez que lo incluyen, y sitúan la Crítica del Juicio en el centro de una consideración original sobre los orígenes de la. modernidad.

Puede abordarse esta perspectiva desde una posición a primera vista más académica: el origen de la disciplina estética. Sabido es que la estética en cuanto disciplina autónoma surge en el siglo xvul. Hasta entonces habia habido reflexiones estéticas - y una historia del pensamiento estético sc remonta siempre a los griegos, mundo en el que se perfilan algunos de los conceptos fundamentales de lo estético y de la disciplina estética--, pero tales pensamientos se producen habitualmente en el marco de teorizaciones más amplias y al margen de una delimitación disciplinar. En el Siglo de las Luces, por el contrario, se asiste a una preocupación especifica por lo estético que atañe incluso a su denominación. El término "estética» no posee todavía el sentido con que actualmente lo entendemos, y en el mismo sistema kantiano se puede apreciar la dificultad de la precisión. En la Crítica de la razón pura "estética" hace referencia al momento primero, intuitivo o sensible del proceso de conocimiento, y tanto en la Critica del Juicio como en cl texto que ya se conoce como Primera introducción a la "Crítica del Juicio", el filósofo se ve obligado a llamar la atención sobre los dos sentidos del término: primer momento del conocimiento y efecto del gusto $o$ análisis de tal efecto estético (estético y estética, respectivamente). ${ }^{1}$

Esta dualidad no es una cuestión meramente terminológica y, a pesar de lo que afirma Kant en el texto citado en la nota, creo que hay algo más que una "carencia de otra expresión" para el uso de «estética». Existe un punto de contacto entre estética (intuición 